\title{
REDES, CULTURA, E IDENTIDAD EN LAS ORGANIZACIONES
}

\author{
Steven B. Andrews
}

Yale University

Carleen R. Basler

Yale University

Xavier Coller

Yale University y Universidad de Barcelona

\begin{abstract}
RESUMEN
La interacción entre cultura y estructura informal de la organización tiene efectos diversos en la vida diaria de las instituciones. Algunos de ellos son el desarrollo de una cierta identidad organizativa y la aparición de relaciones de poder informal. Este artículo avanza varias líneas de investigación sobre estos dos aspectos aprovechando algunas de las aportaciones del análisis de redes. Los autores aventuran algunas hipótesis de trabajo que pueden ayudar a orientar investigaciones empíricas en el futuro.
\end{abstract}

\section{INTRODUCCIÓN ${ }^{1}$}

Un número creciente de trabajos se ocupa del papel que desempeñan las estructuras sociales informales en las organizaciones, de las redes sociales en

1 Los autores agradecen a Alice Andrews, David Knoke y Lynn Smith-Lovin sus comentarios a distintos borradores de este artículo, así como a un evaluador anónimo de la REIS. Roberto Garvía hizo comentarios agudos al borrador en castellano que han ayudado a darle claridad y a mejorarlo. Se presentaron versiones previas de este estudio en la Eastern Sociology Society Annual Meeting en Philadelphia (1998), en la Annual Work-in-Progress Conference, Sociology Department, Yale University (1998), y en el VI Congreso Español de Sociología, A Coruña (1998). Agradecemos a los participantes en estas reuniones intelectuales sus comentarios y críticas. Este trabajo es una adaptación al castellano del artículo "Organizatio- 
las que los actores se ven inmersos, y de los efectos de estos dos elementos en el comportamiento y la actitud de los miembros de la organización. Los/as científicos sociales de diferentes disciplinas suelen coincidir en que la posición de una persona en la estructura social moldea su comportamiento y actitud $^{2}$. Los trabajos pioneros en esta línea estructuralista indican que los fenómenos de carácter normativo como la identidad y la cultura son el reflejo (o la manifestación) de la estructura social subyacente en una organización o grupo.

Varios autores han criticado el enfoque estructuralista argumentando que los aspectos normativos de cualquier organización (como la cultura o la identidad) adquieren vida propia y se independizan de la estructura de la que una vez emergieron (Emirbayer y Goodwin, 1994). Los estudios sobre análisis de redes también toman seriamente la tarea de examinar la cultura no como el reflejo de la estructura social subyacente, sino como un proceso social distintivo y en cierto modo autónomo (Padgett y Ansell, 1993; White, 1992). Otros trabajos se centran en las maneras en que la posición estructural de un individuo limita el desarrollo de sus estrategias de acción en el nivel macro (Johnson y Ford, 1996) y micro (Lawler y Yoon, 1996) ${ }^{3}$. Por otra parte, Gartrell (1987) desafía estas críticas al incorporar fenómenos de la estructura social en las explicaciones sobre la identidad y la cultura ${ }^{4}$.

Dadas las diferentes definiciones que se ofrecen en el mercado de las ciencias sociales sobre la noción de "cultura», y especialmente "cultura de la organización», no se puede más que reconocer con DiMaggio (1994) que se trata de un término resbaladizo y ambiguo. Aunque el concepto de «cultura de la organización» está infrautilizado, algunos autores lo encuentran bastante útil para entender el comportamiento tanto de la organización como de sus miembros (Schein, 1985, 1996) . Esta perspectiva indica que la cultura determina el comportamiento, de la misma manera que los estructuralistas indican que para

nal structures, cultures and identities. Overlaps and differences», aparecido en Research in the Sociology of Organizations, vol. 16, pp. 213-235. Los autores desean agradecer una primera traducción de Gemma Sala. La adaptación del texto para la comunidad sociológica hispanohablante es responsabilidad del tercer autor, quien agradece al Centro de Estudios Avanzados en Ciencias Sociales que haya puesto a su disposición su biblioteca, y a Jesús M. de Miguel sus anotaciones al artículo.

${ }^{2}$ En realidad, esta visión es compartida tanto por el marxismo como por el funcionalismo. La teoría de la estructuración, además, abunda en la idea de que el comportamiento de las personas contribuye a reproducir la misma estructura social que determina su comportamiento. A este respecto, véase el texto de Giddens (1984). Véase el trabajo de Burke (1997) para una perspectiva diferente.

3 Ésta es la postura básica de la teoría de la estructuración desarrollada por Giddens (1984).

${ }^{4}$ Los trabajos sobre identidad están abandonando el enfoque microsociológico basado en la interacción para incorporar los efectos construccionistas de aquellos elementos que, como la raza o el género, suelen caracterizar a grupos de individuos (Cerulo, 1997).

5 Se puede consultar el trabajo de Garmendia (1988) para una primera aproximación en castellano a los debates sobre el concepto de cultura de la organización. 
comprender el comportamiento de la organización es suficiente y necesario un buen conocimiento de su estructura.

La perspectiva estructuralista y la culturalista se pueden combinar fructíferamente para la investigación. Por un lado, se sabe que la cultura y la estructura social informal están interrelacionadas. Ambas son entendidas desde el punto de vista de la teoría de la estructuración como el resultado de la estructura de un grupo u organización y como elementos que facilitan la tarea de formación de la estructura social. Por otro lado, la cultura de la organización y la estructura social informal, independientemente o de forma combinada, afectan a los miembros de la organización: moldean sus identidades organizativas y se convierten en una limitación o una oportunidad para la realización de sus tareas. En la sección siguiente desarrollamos el concepto de cultura aplicado a las organizaciones.

\section{CULTURA DE LA ORGANIZACIÓN}

La sociología, antropología y la psicología social suelen presentar la cultura como el componente principal del funcionamiento de la sociedad. Los sociólogos/as tienen un antecedente de primera magnitud en Durkheim y en el funcionalismo parsoniano. Los/as autores "culturalistas» tienden a concebir la cultura como un aspecto relevante en la adaptación de las organizaciones y la suelen definir como el sistema normativo de pautas de comportamiento que se transmiten socialmente y que vinculan a los actores con sus entornos ${ }^{6}$. En este artículo nos centramos en la cultura de las organizaciones. Schein (1990: iii) define la cultura de la organización como «un (a) conjunto de asunciones básicas, (b) inventadas, descubiertas, o desarrolladas por un grupo determinado (c) en el proceso de aprendizaje que se desarrolla al enfrentarse con problemas de adaptación externa e integración interna [de la organización], (d) que han funcionado lo suficientemente bien como para considerarlas válidas y, por tanto, (e) se enseñarán a los miembros nuevos como la (f) manera correcta de percibir, pensar, y sentir en relación con estos problemas [de integración y adaptación]». Schein entiende la cultura organizacional como un mecanismo de defensa colectiva por el que los miembros de una organización aprenden a enfrentarse a los problemas derivados de los desafíos internos y externos a la organización.

Para esta perspectiva, la cultura es vista como el principal determinante del comportamiento: "el nivel más profundo de la cultura hace referencia a lo cognitivo; las percepciones, el lenguaje, el proceso de pensamiento que un grupo comparte es la causa determinante de los sentimientos, actitudes, valores, y

${ }^{6}$ Véase el trabajo de Keesing (1974) para una revisión de las concepciones diversas de la noción de cultura en las ciencias sociales. 
comportamiento»(Schein, 1990: iii). En esta concepción de la cultura de la organización se condensan dos perspectivas sociológicas de amplio raigambre: la escuela funcionalista y de la sociología fenomenológica. Para algunas personas, la cultura se concibe como un atributo que mantiene el orden social. Es concebida, por tanto, como algo externo al individuo. Para otras personas, la cultura es como un sistema de reglas internalizado que guía el comportamiento de los individuos ${ }^{7}$. Cada perspectiva enfatiza niveles y unidades de análisis diferentes.

El primer grupo de teorías intenta comprender la cultura de un grupo o subgrupo, las funciones que desempeña en el mantenimiento del grupo, o las condiciones en las que se desarrolla el grupo y su cultura (Evered, 1983). Los trabajos que se encuadran en este enfoque intentan describir el objetivo a que sirven y las funciones que desempeñan las creencias y símbolos en las organizaciones, presentándolos ambos como elementos necesarios para el orden y la estabilidad social (Ott, 1989). Gran parte de estos trabajos se centra en analizar las maneras en que la cultura puede servir como elemento integrador de los individuos en la organización (Trice y Beyer, 1984) o como instrumento de control interno (Wilkins y Ouchi, 1983). Su naturaleza cambiante, evolutiva, permite a estos autores concebir la cultura como un elemento resistente a cualquier intento de manipulación.

El segundo grupo de teorías concibe la cultura como algo que reside dentro de cada persona $y$, por tanto, se entiende que se puede analizar a través del estudio de los procesos de aprendizaje, construcción, y atribución de significados y causas ${ }^{8}$. La cultura es algo que los miembros de una organización aprenden y, por tanto, habitualmente produce creencias compartidas. Desde esta perspectiva, la cultura se concibe como un elemento abierto al cambio o a la variación ya que las personas no la procesan e incorporan de manera idéntica. En el presente artículo entendemos que la cultura es un elemento de la organización al que los individuos acceden de forma diferente.

Diversos trabajos - especialmente los de inspiración empresarial o los que se utilizan en las escuelas de negocios_ discuten sobre «la cultura» de una organización. Se asume que cada organización ha desarrollado a lo largo del tiempo una cultura determinada que la hace distinta y que en parte explica la situación de la empresa en el mercado. En otros trabajos se examina el desarrollo de subculturas dentro de una misma organización ${ }^{9}$. Por ejemplo, Van Maanen y Barley (1984) defienden que es más probable que los miembros de un grupo ocupacional desarrollen valores y creencias compartidas que los miembros de una organización que están dispersos espacialmente y que desa-

7 Sobre el papel de la cultura en la sociedad según la perspectiva funcionalista, véase el trabajo de Parsons (1966). Para la perspectiva de la sociología fenomenológica, véase el trabajo de Berger y Luckmann (1986).

8 Véanse los trabajos de Gioia y Thomas (1996), Harris (1994) y Levitt y March (1988).

9 Véanse los trabajos de Gregory (1983) y Martin y Shiehl (1983). 
rrollan funciones diferentes en la estructura de la organización ${ }^{10}$. Los miembros de una planta de producción o una sucursal bancaria pueden desarrollar una cultura local que puede ser diferente a la de otras plantas o sucursales dentro de la misma organización ${ }^{11}$. El fenómeno puede tener lugar incluso entre grupos de una misma planta o sucursal. La cultura local emergente puede ignorar e incluso transgredir las metas que la organización intenta alcanzar $^{12}$.

Uno de los centros de interés de ciertos sociólogos/as ha sido el efecto de la cultura de la organización en el rendimiento de la misma o de sus miembros. En el plano individual, parece existir una asociación entre la cultura organizacional y la moral y participación de los miembros (Allcorn, 1995). En el plano de la organización, parece existir una asociación entre cultura y éxito (Kotter y Heskett, 1992). Una organización que fomenta una cultura que valora los recursos humanos suele estar mejor preparada para enfrentarse a (y sobrevivir en) un entorno que se torna turbulento e incierto (Welbourne y Andrews, 1996). En consecuencia, la cultura de una organización puede convertirse en un activo empresarial que produzca pingües beneficios, y no sólo económicos (Kotter y Heskett, 1992; Garmendia, 1994).

Este tipo de trabajos suele asumir una relación estrecha entre cultura y rendimiento de la organización. Sin embargo, Pfeffer (1981) sugiere que ambas variables no están relacionadas ya que los resultados de las organizaciones en parte dependen de los recursos y oportunidades del entorno y de su utilización, no necesariamente de la cultura. Es más, aunque los/as investigadores en organizaciones suelen hablar de la relación entre cultura y funcionamiento de la organización, muy raramente desarrollan explicaciones teóricas u ofrecen pruebas que apoyen sus tesis (Siehl y Martin, 1990) ${ }^{13}$.

Algunos investigadores/as se han centrado en la fuerza e intensidad de la cultura en la organización. Diferencian entre culturas fuertes y débiles. La asunción suele ser que las culturas que están fuertemente incorporadas en la vida de la organización y de sus miembros producen un mejor rendimiento (en el plano colectivo e individual), facilitan que se hagan sacrificios personales en aras de la organización, y promueven una mayor y más sólida identificación de los miembros con la organización ${ }^{14}$. El peligro de las culturas sólidas e integradas es que pueden cegar a las personas en su percepción de la

10 Pueden verse algunos ejemplos en el trabajo de Burawoy (1986).

11 Véase el trabajo de Edwards et al. (1999) y el de Coller (1996) para el caso de las multinacionales y el papel de la cultura local en la dirección de las subsidiarias.

12 Sobre los conflictos entre cultura local y objetivos de las organizaciones la escuela institucionalista ha hecho aportaciones básicas. Véanse los trabajos de Gouldner (1954) y Selznick (1949). Para un caso español se puede ver el trabajo de Garvía (1997: 77-180), en el que se analiza la evolución de la cultura de la ONCE, la transformación de los objetivos de esta organización y los conflictos que se generan.

13 Una excepción es el trabajo de Denison y Mishra (1995).

14 A este respecto, véase el trabajo de Deal y Kennedy (1982) y el de Hofstede (1980). 
realidad para tomar decisiones sobre aspectos relevantes para la organización. Por contra, las culturas que están más débilmente implantadas (o asumidas, incorporadas) suelen ser también más flexibles porque los individuos incorporan elementos culturales de otros sitios. Aunque las culturas débiles pueden garantizar una mayor independencia de criterio, por esto mismo pueden ser también fuente de conflicto al generar una mayor ambigüedad ${ }^{15}$. Una cultura que no sirva de base para respuestas claras y compartidas a los desafíos del entorno (es decir, una cultura débil) aumenta la probabilidad de que los individuos recurran a sus propias experiencias, atribución de significados, mitos (independientemente de la organización) para elaborar estas respuestas. De esta manera se desarrollarán soluciones contradictorias que pueden ser fuente de conflictos.

Kotter y Heskett (1992) apuntan que el debate sobre la cuestión de la solidez e implantación de una cultura de la organización puede haber adquirido una orientación errónea, al menos cuando los/as investigadores se ocupan de las consecuencias sobre el rendimiento. En su lugar, estos autores se centran en el grado de adaptabilidad de una cultura. Indican que se debería averiguar si la cultura de una organización sirve para homogeneizar y motivar a los miembros, y si es funcional para que los miembros sean capaces de dar respuestas adecuadas a las presiones y demandas del entorno. Una cultura sólida, ampliamente incorporada en la vida organizativa, pero no adaptativa, puede cohesionar a los miembros de la organización, pero conducirles hacia soluciones erróneas o inadecuadas ante problemas planteados desde el entorno.

Aunque parece claro que no se han desarrollado muchos conceptos compartidos y aceptados en el área de la cultura organizacional, conviene resaltar algunos aspectos.

Primero, la cultura de una organización consiste en el conjunto de asunciones más o menos compartido por sus miembros. Se manifiesta en rituales, símbolos, normas y lenguaje. Genera sus propios mitos. La cultura define el tipo de comportamiento que es apropiado en una organización. De esta manera, la cultura de una organización sirve para integrar a sus miembros en el colectivo. Es decir, cumple la función de servir de soporte para crear identidades organizacionales en la medida en que cada miembro internaliza los componentes esenciales de una cultura particular. Estos componentes se suelen internalizar de manera diferenciada por cada miembro, quien accede a la cultura de una forma fragmentaria (DiMaggio, 1997). Por este motivo, es previsible que la identidad organizacional no sea homogénea.

Segundo, el cambio en las organizaciones, cualquiera que sea su fuente, puede ser disruptivo y amenazador. Cuando se intenta introducir algún cam-

15 Se ha estudiado poco el tema del conflicto cultural en las organizaciones desde un punto de vista sociológico. Una aproximación es el trabajo de Gregory (1983). Se trata de una de las áreas con más potencial debido a los cambios continuos en las empresas que afectan a la manera en que sus miembros perciben e interpretan la realidad organizativa en la que están inmersos. 
bio (porque así se ha decidido o por culpa de factores que se escapan al control de la organización), la cultura puede ejercer una función de barrera cuando el cambio es incompatible con la cultura que ya existe. Aunque las personas pueden recurrir a la cultura existente como un mecanismo de defensa, el cambio puede amenazar y erosionar esta cultura.

Tercero, la cultura de la organización es un elemento que es muy difícil de medir $^{16}$. El desacuerdo que existe entre los/as científicos sociales sobre los elementos constitutivos de la cultura incrementa esta dificultad. En particular, la manera en que la cultura de una organización se institucionaliza es algo que permanece menos claro de lo que sería deseable. A pesar de los esfuerzos de la escuela neoinstitucionalista por comprender la cultura como algo dinámico, los factores que explican la creación y mantenimiento de una cultura, o su transformación, no están del todo claros.

Dada la raíz interaccionista de muchos de estos trabajos, una variable explicativa del proceso de institucionalización que posee cierto potencial es la estructura de la organización, tanto en sus aspectos formales como - y quizá más importantes - los informales. DiMaggio (1992) indica que el tono y el estilo de una organización (su cultura) puede predecirse por la combinación de diferentes posiciones en la red de relaciones sociales (estructura informal) y de posiciones en el sistema formal de papeles sociales (estructura formal). El autor pone poco énfasis en el papel de los atributos personales. DiMaggio otorga la primacía a la estructura informal porque facilita la acción y media entre los atributos de un actor y la posición formal en lo que se refiere a los resultados de la organización. La estructura, además de los rasgos de la personalidad, pueden explicar por qué la cultura fluye de forma desigual entre los miembros de la organización.

Antes de discutir cómo funciona esta asociación conviene prestar atención a las redes sociales. Dada la amplitud del análisis de redes, no se intentará revisar todos los aspectos de este enfoque. Nuestro objetivo es poner de relieve algunos conceptos básicos ${ }^{17}$.

${ }^{16}$ Las cuestiones y los métodos de investigación sobre cultura de la organización se han discutido en varias ocasiones. Véanse los trabajos de Burrell y Morgan (1979), Ouchi y Wilkins (1985) y Smircich (1983).

${ }_{17}$ Para una visión excelente en castellano del análisis de redes, véase el trabajo de Josep A. Rodríguez (1995) y su aplicación práctica en su artículo sobre «la sociología académica» (1993). Véanse también los trabajos de Félix Requena (1989, 1996). Los textos de Wasserman y Faust (1994), Nohria y Eccles (1992), Powell y Smith-Doerr (1994) son básicos. Los trabajos sobre redes están en un momento de transformación. En los últimos tiempos se decantan por el estudio de cómo las redes cambian con el tiempo, y cómo se producen los efectos de retroalimentación (Suitor, Wellman, y Morgan, 1997). Esto implica que la asociación entre posición y resultados, como la formación de identidad, no es unidireccional ni lineal. 


\section{REDES INFORMALES EN LA ORGANIZACIÓN}

Existen dos tradiciones en el estudio de las redes informales en las organizaciones. Una se centra en la manera en que las redes influyen sobre la efectividad de la organización y entronca con los estudios de Elton Mayo y de la escuela de relaciones humanas. En esta tradición, la estructura informal es vista como un freno para el desarrollo de la organización (ya que contribuye a subvertir, contradecir, o resistirse a las órdenes de la dirección), o como un multiplicador de la productividad del trabajador/a al contribuir a crear un ambiente favorable. La otra se dedica a analizar cómo las redes sociales informales suelen ser utilizadas para evitar las rigideces de la estructura formal. Esta segunda tradición entronca con los estudios de Gouldner (1954) y Burawoy (1986), y en ella se incluyen los autores que perciben la estructura informal como un elemento que de forma inesperada facilita la consecución de los objetivos organizacionales gracias a que suaviza las relaciones entre los miembros de distintos niveles jerárquicos y crea confianza entre miembros que están distantes en la jerarquía de la organización ${ }^{18}$.

Estas dos tradiciones se complementan una a otra, aunque no está claro qué aspectos de la estructura son más importantes. Hay tres elementos que se suelen citar como los más relevantes para el comportamiento de los actores en las organizaciones: proximidad, equivalencia estructural y centralidad. La influencia social que facilita el contacto directo (o proximidad) entre las personas en un grupo cohesionado puede contribuir a desarrollar percepciones y juicios similares respecto de su entorno inmediato (Meyer, 1994). La participación en un grupo genera el refuerzo de las normas y la convergencia de los juicios personales acerca de la realidad, especialmente en redes sociales de carácter afectivo ${ }^{19}$.

La equivalencia estructural de los actores puede también conducir a la adopción de puntos de vista, actitudes y comportamientos similares (Burt, 1980). Los actores que ocupan posiciones estructuralmente equivalentes en una organización se enfrentan a presiones y limitaciones similares que derivan de su relación relativamente parecida respecto de otros actores y, por tanto, suelen desarrollar percepciones del entorno e identidades similares, incluso sin tener contacto directo entre ellos.

Los actores que ocupan posiciones centrales suelen estar bien situados para

18 Véase el trabajo de Coller (1997) para un estudio de caso en España en el que se analizan las estrategias utilizadas por los actores para soslayar la estructura formal en momentos de turbulencia y cambio. Véase el trabajo de Ortiz (2000) para un análisis comparado del recurso a la estructura formal como instrumento de negociación de los cambios en la organización.

19 Véanse los trabajos de Uheara (1990) y de Ibarra y Andrews (1993). La psicología social ha avanzado enormemente en el análisis del desarrollo de las percepciones del mundo similares que elaboramos las personas y de las variables más significativas que intervienen en este proceso. Véase a este respecto el texto clásico de Aronson (2000: 31-67, 119-173). 
influir sobre las percepciones que tienen otros actores de la realidad inmediata a través de dos mecanismos: influencia social y control del flujo informativo (Brass y Bukhardt, 1992; Cook y Emerson, 1984). Mientras los actores que son estructuralmente equivalentes suelen experimentar el mismo proceso de formación de la identidad organizacional, los actores centrales en una red social tienen la capacidad de influir en las actitudes e identidades de otros actores en posiciones no equivalentes. El efecto de la centralidad en la influencia social suele depender del tipo de la relación entre actores. En general, la influencia suele ser más relevante en relaciones de tipo instrumental que en las afectivas (Ibarra y Andrews, 1993).

En la medida en que las interacciones de carácter informal entre los miembros de una red están regidas por principios afectivos (homofilia), estas interacciones pueden funcionar como un contrapeso a los principios universalistas que suelen orientar las acciones de la burocracia de cualquier organización $^{20}$. Las redes de carácter expresivo en las que existe un componente fuerte de afectividad (redes de amistad, familiares, de ayuda mutua, en una organización) tienden a estar gobernadas por relaciones de tipo homofílico, lo cual refuerza los valores y percepciones comunes a los miembros de la red y, por tanto, favorece la conformidad (Ibarra, 1992a).

Cuando los miembros de un grupo tienen limitaciones estructurales para acceder a recursos e información valiosos, tal como ocurre con actores marginales en organizaciones jerarquizadas, la homofilia se convierte en perjudicial ya que puede reforzar o conducir a la marginalidad (Ibarra, 1992b). Los vínculos afectivos entre miembros de una organización tienden a ser vínculos fuertes y robustos. Los vínculos fuertes fomentan y aseguran el acceso a relaciones de solidaridad, mientras que los vínculos débiles proveen de información y recursos más allá de los que están disponibles en el círculo de acción de un actor (Burt, 1992; Granovetter, 1973). Los vínculos débiles son fuertes en la difusión de innovaciones y de información, mientras que los vínculos fuertes entre los miembros de un grupo o red contribuyen a la cohesión del grupo.

Hay dos grupos de ideas que son relevantes para la discusión que se inicia más abajo. Por un lado, están los conceptos que hacen referencia a los mecanismos por los cuales las redes sociales limitan la acción de los individuos en una organización: proximidad, equivalencia, y centralidad en la red. Por otro lado, se encuentra el grado en que el vínculo entre personas de la red promueve una cierta cohesión grupal. Aquí la bibliografía se centra en las nociones de fortaleza del vínculo y homofilia (afectividad). La diversidad en

20 La tensión entre universalismo y particularismo arranca del análisis que hace Weber de la burocracia (1978: 956-1002) y que posteriormente recoge Parsons (1959: 360, 412-15) en sus pares contrapuestos. Sobre los principios homofílicos basados en relaciones afectivas, véanse los trabajos de Brass y Bukhardt (1992), Ibarra (1992b) y Heimer (1992). Sobre el efecto de este tipo de relaciones en la universidad española, véase el trabajo de Pérez-Díaz y Rodríguez (2001: 138 ss.) y el de Jesús M. de Miguel (2002: 1191 ss.). 
la red puede ocurrir en cualquiera de las dimensiones: fortaleza u homofilia. Una vez revisados los conceptos básicos de la cultura organizativa y de las redes en la organización, conviene prestar atención ahora a la interacción entre estas dos esferas.

\section{CULTURA, ESTRUCTURA, E IDENTIDAD}

Conviene examinar las maneras en las que los elementos de la organización interactúan, concretamente la cultura y la estructura informal. Esta interacción puede que influya en la creación y consolidación de la cultura organizativa. La emergencia de identidades organizativas debería ser más visible en momentos extraordinarios (en el sentido durkheimiano), no rutinarios y envueltos en cierto grado de incertidumbre tal como son los reveses que el entorno provoca a la organización (Meyer, 1982). Estos momentos implican cambios que ponen en cuestión la naturaleza de las rutinas e interacciones diarias que se suelen dar por supuestas (Elsbach y Kramer, 1996). La manera en que los miembros de la organización deciden buscar ayuda, en esos tiempos turbulentos, y el proceso por el cual obtienen esta ayuda, pueden estar influidos por la cultura y la estructura informal de la organización. Por ejemplo, no es lo mismo buscar ayuda en una red gobernada por principios afectivos que en otra de carácter universalista o jerárquico. La cultura de una organización puede fomentar una u otra opción, como en el caso de culturas en las que se enfatiza el apoyo social y la cooperación, o el individualismo. Proponemos una agenda de investigación diseñada para conocer mejor los ámbitos en los que la superposición de estas dos áreas de investigación pueden iluminar aspectos la una de la otra.

En el plano más general sugerimos que, al menos en el contexto organizativo, se tenga en cuenta que la cultura y la estructura son fenómenos relacionados y que se influyen mutuamente de forma continua. Ninguna domina todo el tiempo. Tampoco ninguna es el mero reflejo de la otra. Por ejemplo, la cultura organizativa en parte guía las elecciones que hacemos respecto de con quién decidimos interactuar. Nuestras interacciones afectan a los flujos de información que ayudan a construir las estructuras normativas (que nos dicen lo que se puede y no se puede hacer, lo que es conveniente y lo que no) que creamos para nosotros mismos al tiempo que internalizamos la cultura organizativa. Los culturalistas o estructuralistas que obvian el funcionamiento de la otra área corren el riesgo de malinterpretar el funcionamiento del mundo social real.

Analizar la interacción entre la estructura y la cultura organizativa desde un punto de vista teórico no es suficiente ni útil ya que se trata de una cuestión de carácter empírico. Como tal, ofrecemos algunas proposiciones que se pueden contrastar en investigaciones futuras. En la medida en que estas propo- 
siciones no están todavía comprobadas, la utilidad de nuestro argumento permanece inédita. Para contribuir a la resolución de este asunto sugerimos situaciones potenciales en las que se pueden poner a prueba nuestras proposiciones. En primer lugar, discutimos las maneras en que la cultura y la estructura interaccionan y generan identidades organizativas. Posteriormente, discutimos las áreas potenciales de investigación.

\section{IDENTIDAD ORGANIZACIONAL}

Es un lugar común en la sociología de las organizaciones indicar que las identidades tienen dos componentes: personal y organizativo (Ashforth y Mael, 1989) ${ }^{21}$. El componente personal es idiosincrásico y exógeno en relación al desarrollo de una cultura local en la organización. El componente organizativo de la identidad suele ser más uniforme entre los miembros y puede llegar a dominar la identidad personal. En este trabajo entendemos que la identidad organizativa es una identidad colectiva; esto es, la representación de la organización compartida por sus miembros o por grupos de ellos $^{22}$. Creemos que la identidad organizacional es un derivado de la cultura de las organizaciones tamizada por el filtro cognitivo de cada individuo. En consecuencia, las identidades organizativas no son necesariamente consensuadas, sino que pueden ser fuente de conflictos y existir varias no siempre de forma complementaria. Las identidades organizativas son importantes. Se ha demostrado que moldean la forma en que las personas procesan la información y que afectan tanto a su satisfacción como a la efectividad de la organización $^{23}$.

Las identidades organizativas son referentes culturales construidos vía socialización e interacciones. Esto quiere decir que el grado de implantación (y ciertamente su existencia) variará. Las redes sociales desempeñan un papel crucial en este proceso de construcción. Por un lado, limitan pero, al mismo tiempo, promueven las interacciones a través de las cuales se suele construir la identidad organizativa (DiMaggio, 1992). La participación en las redes sociales

${ }^{21}$ El uso que hacemos del concepto de identidad es menos amplio que el de yo (self): los constructivistas sociales consideran que el yo está construido a partir de identidades socialmente situadas. Hay dos comentarios al respecto. Por un lado, si bien la cultura se entiende como un atributo de la organización, las identidades organizativas son un fenómeno individual, y puede cambiar de una persona a otra. Por otro lado, un individuo puede tener varias identidades a la vez, y no siempre ser capaz de armonizarlas.

${ }_{22}$ Esta concepción de la identidad organizativa abre la puerta a considerar que dentro de una misma organización pueden coexistir identidades diferentes, no necesariamente en conflicto o competición, pero tampoco necesariamente complementarias.

${ }_{23}$ A este respecto, véanse los trabajos de Baron y Pfeffer (1994) y Diamond (1993). Las identidades organizativas son la base sobre la que puede asentarse lo que Jesús M. de Miguel (1990: 75 ss.) llama el "poder invisible» de las organizaciones, ya que conducen a las personas a actuar de manera favorable a la organización. 
genera las situaciones en las que se desarrollan las interacciones entre las personas. Las interacciones no ocurren al azar ya que hay dos factores que limitan la construcción de vínculos en las organizaciones. El primero es la estructura formal, que hace que la creación de ciertos vínculos sea más o menos probable según la situación en la jerarquía y que superpone las relaciones de poder a los vínculos existentes. El segundo es la existencia de la oportunidad de interaccionar con aquellas personas que estén disponibles para iniciar una relación.

La estructura formal y la informal en organizaciones jerarquizadas están emparejadas de forma laxa. Mientras las estructuras normativas formales pueden moldear una red emergente, Krackhardt (1992) indica que los vínculos fuertes que se extienden a través de distintos niveles jerárquicos generan la confianza suficiente para que los actores cooperen bajo condiciones de cambio e incerteza. Esto sugiere que allí donde estas relaciones se hallen presentes se puede desarrollar una cultura de relaciones cooperativas y de resolución conjunta de problemas. Aunque los vínculos débiles pueden canalizar elementos que supongan variaciones en la identidad organizativa, los vínculos fuertes basados en el contacto personal pueden constituir los elementos fundamentales de esta identidad. Pero los vínculos fuertes también moldean la cultura emergente.

En la medida en que la identidad organizacional es el resultado de interacciones sociales, es también el producto de procesos de influencia social. La dinámica de las redes sociales puede tener un efecto homogeneizador en la formación de la identidad, de manera que no es extraño observar la creación de identidades similares en un conjunto de actores que participan en una o varias redes interconectadas. Por ejemplo, los actores que son estructuralmente equivalentes pueden desarrollar identidades similares ya que por su posición están expuestos a presiones parecidas y se enfrentan con opciones similares. De la misma manera, los actores que están próximos pueden también converger hacia identidades parecidas ya que están expuestos a flujos de información e influencias sociales similares. La centralidad puede reforzar también una identidad organizativa determinada (Dutton, Dukerich y Harquail, 1994). Los actores centrales de una red pueden incorporar de una manera más directa y sólida la identidad organizativa en su psique. La centralidad puede ayudar a que las personas que ocupan posiciones centrales en una estructura social informal se forjen una identidad organizativa compartida, similar, que puede ser distinta de la identidad que se construyan los miembros de la periferia de una red. Entre los actores periféricos puede que la dimensión personal de la identidad organizativa sea dominante. De aquí derivamos la primera proposición.

Proposición 1A. La identidad organizacional construida por cada persona depende en parte de su posición en la estructura formal e informal de la organización. Quienes están sujetos a influencia social por proximidad o por equivalencia deberían tener identidades similares. La proximidad debería dar lugar 
a identidades parecidas entre personas que pertenecen a grupos cohesionados. La equivalencia supuestamente conecta identidades entre grupos.

Si las identidades organizacionales dependen en parte de la centralidad de los individuos en la red, y la centralidad varía en función del acceso y el control de recursos, es previsible que exista un amplio repertorio de identidades allí donde haya una diferencia significativa de poder en una organización. Por el contrario, cuando el desequilibrio de poder es menor (por ejemplo, allí donde haya pocos niveles jerárquicos), el repertorio de identidades se reducirá y éstas tenderán a converger. Sin embargo, estos efectos de la centralidad se confunden en ocasiones con las preferencias homofílicas. En la medida que la posición en la estructura puede dificultar el acceso a los actores centrales, la homofilia puede dar lugar a identidades organizacionales marginales (Ibarra, 1992b), que incluso pueden ser de oposición a las normas generales de la organización. En relación a las preferencias homofílicas, la fuerza de los vínculos en una red puede ser relevante para la formación de identidades. Los vínculos débiles pueden convertirse en canales a través de los cuales se incorporan elementos diversos, contradictorios a veces, en el proceso de construcción de la identidad organizacional. De esta forma, contribuyen a debilitar la consistencia de la identidad entre los miembros de la organización. Por contra, los vínculos fuertes que caracterizan a los grupos cohesionados pueden reforzar la identidad común. De estas reflexiones se deriva la proposición siguiente.

Proposicion 1B. Cuanto mayor sea el rango de la centralidad informal y/o de estructura jerárquica formal, más amplio será el abanico de identidades formadas.

Aquellas personas que disponen de más recursos sociales y de una mayor variedad de flujos de información suelen estar mejor situadas para influir en la configuración de las identidades de otros y para resistir la influencia de otros en su propia identidad. Sin embargo, la identidad también determina el acceso a unas posiciones u otras. En la estructura formal, las personas cuya identidad organizacional es consistente con la cultura de la organización tienen más posibilidades de ser promocionadas (Podolny y Baron, 1997). Pero esta correspondencia no se mantiene en el ámbito de las redes informales. En este ámbito, el acceso a los recursos sociales y a la información (es decir, una posición central) debería llevar a la formación de una identidad bastante fuerte. Una identidad fuerte o sólida implica la asimilación de los objetivos de la organización y los comportamientos sancionados para alcanzar tales objetivos. También implica que la persona ha internalizado el conjunto de expectativas que se tiene sobre ella y actúa consecuentemente.

Por el contrario, un acceso limitado a los recursos de la organización y a los flujos de información debería conducir a una identidad débil e inestable que puede ser fácilmente sustituida por otra. Asimismo, la disonancia entre grupos puede dar lugar a identidades débiles. Los lazos interpersonales propor- 
cionan referentes significativos para la construcción de identidades (Gartrell, 1987; Powell y Brantley, 1992). Sin embargo, cuando las personas se enfrentan a referentes contradictorios, lo más probable es la inactividad o incluso la evasión (Krackhardt, 1992). Esta alienación originada en la disonancia en la red social puede debilitar la identidad organizacional compartida y, consecuentemente, conducir a la formación de identidades más heterogéneas.

Proposición 1C. Para cada miembro de la organización, la diversidad de la estructural informal y la centralidad debería dar lugar al desarrollo y mantenimiento de una identidad organizacional estable. La diversidad se refleja en la heterofilia y en la presencia de agujeros estructurales y de vínculos débiles. La diversidad debería conducir a identidades culturales diferentes entre los miembros, mientras que una posición de centralidad parecida lleva a identidades más uniformes. Sin embargo, si existen presiones contradictorias insolubles en la red, el resultado más probable es el abandono o la retirada (alienación del entorno) y, consecuentemente, emergerán identidades organizacionales débiles.

Estas primeras proposiciones sugieren que en cierta medida la identidad es una función de la estructura. Pero aún no se han tenido en cuenta los efectos de la cultura de la organización en la creación y en el mantenimiento de identidades organizacionales. La cultura y la identidad están relacionadas. Por ejemplo, algunos autores consideran que la cultura de la organización se apoya en la identidad organizacional del mismo modo que la consciencia se fundamenta en lo subconsciente (Diamond, 1991). Los contactos en la red sirven para canalizar costumbres, valores, actitudes y comportamientos estándar (Gartrell, 1987), elementos todos ellos que suelen ser considerados la esencia de la cultura de la organización. La adopción de una identidad organizacional es en parte el resultado de la absorción de esos elementos de la cultura.

La identidad organizacional también afecta a la manera en que los miembros de la organización se enfrentan a las presiones internas y externas (Elsbach y Kramer, 1996). Cuando la cultura de la organización es fuerte (homogénea) y unitaria, independientemente de si además es una cultura adaptativa, es esperable una tendencia notable hacia la conformidad. Las personas incorporadas a culturas fuertes pueden sentir una presión mayor para adecuarse a lo establecido. Como también es cierto que los inconformistas suelen tener más probabilidades de ser separados de la organización, podemos esperar de las culturas fuertes que promuevan identidades organizacionales estables y parecidas.

Proposición 1D. Cuanto más fuerte sea la cultura local, más parecidas serán las identidades organizacionales entre los miembros. Estas identidades deberían ser uniformes, siempre y cuando sean también estables. La (dis)funcionalidad de estas identidades depende de si la cultura local es adaptativa o no. La existencia de subculturas fuertes debería dar lugar a identidades diferenciadas y potencialmente en oposición a la dominante.

Estas proposiciones sugieren que las identidades estables emergen en cultu- 
ras fuertes donde hay influencias sociales estables. Sin embargo, es más probable que las culturas fuertes se construyan en condiciones de homogeneidad estructural (por ejemplo, donde existen vínculos fuertes, a través de los cuales circulan informaciones parecidas, con una división débil en cliques). La diversidad estructural (o heterogeneidad) suele ser proclive a la aparición de cliques, subgrupos, cada uno de ellos con una tendencia a generar una subcultura propia. Cuanto más pequeño, homogéneo y denso en vínculos es un grupo, más fuerte será la cultura local. Esto significa que inevitablemente se produce una tensión entre los tipos de cultura y estructura que facilita el desarrollo de identidades organizacionales estables.

Proposición 1E. Las identidades fuertes, estables y diversificadas surgen de la influencia social y de la interacción en la red. Las identidades fuertes y homogéneas resultan de culturas organizacionales fuertes. Existe una tensión entre la existencia de diversas redes y la cultura local: cuanto mayor es la diversidad, más débil es la cultura de la organización o más probabilidad hay de la emergencia de subculturas. Las subculturas más pequeñas pueden dominar la identidad de sus miembros, lo que puede derivar en compartimentos estancos en la organización.

No está clara la manera en que la uniformidad de identidades de una organización afecta a resultados tales como la productividad o la moral. Por ejemplo, la variedad de identidades puede facilitar la innovación o la flexibilidad, pero puede también debilitar la solidaridad. Se espera que la reacción de los miembros de la organización al cambio, por ejemplo, dependa de la fuente de identidades estables. Las culturas fuertes sugieren inercia y resistencia al cambio, mientras que la diversidad estructural implica gran flexibilidad, mejor acceso a la información y los recursos necesarios, y una mayor voluntad para adoptar métodos nuevos o normas.

Proposición 1F. Cuando existen identidades organizacionales estables, la capacidad para reaccionar con éxito a cambios como reestructuraciones o fusiones depende de si la fuente de esas identidades es cultural o estructural. Las identidades basadas en la cultura organizacional deberían resistirse al cambio, mientras que las identidades que tienen una base estructural deberían estar más predispuestas a aceptarlo.

Este efecto no es tan claro como aquí se sugiere. Por ejemplo, una cultura fuerte puede predisponer al sacrificio y a la abnegación para enfrentarse a situaciones traumáticas, mientras que la diversidad estructural puede hacer que los miembros de una organización sean conscientes y aprecien las oportunidades que se les ofrecen fuera de la organización y, por tanto, sean más proclives a abandonarla. Sin duda, cuando la organización responde a un cambio, las identidades de origen cultural (que suelen ser más uniformes) pueden conducir a una aceptación mejor de esa respuesta. Asimismo, cuanto más variadas sean las identidades estructurales, mayor se espera que sea la resistencia a la estrate- 
gia de respuesta al cambio llevada a cabo por la organización. De acuerdo con el modelo de los umbrales de Granovetter (1978), los miembros de la organización deberían presentar umbrales consistentes (ya sean altos o bajos) y más o menos uniformes en lo que respecta a los motivos para la aceptación de nuevas políticas frente al cambio. Por contra, la variedad estructural requiere un abanico más amplio de estos umbrales y, en consecuencia, de motivos.

Proposición 1G. La amplitud con la que los miembros de una organización aceptan y adoptan las estrategias puestas en marcha como respuesta al cambio depende de la fuente de la identidad. La uniformidad de las identidades de origen cultural suele conducir a una adopción de esas estrategias más fuerte y más rápida, mientras que la variación inherente a las identidades de origen estructural supone una adopción más superficial y una mayor resistencia a las políticas del cambio.

\section{PODER}

El poder es un área de interés crucial para científicos sociales, y en concreto para los/as sociólogos de las organizaciones. Muchos trabajos en este área enfatizan los aspectos estructurales, especialmente los derivados de las teorías del intecambio (Cook y Whitmeyer, 1992) o de los enfoques basados en la dependencia de recursos (Pfeffer y Salancik, 1978). Algún trabajo ha destacado el papel de lo que podría llamarse «influencias normativas», como la autoridad y legitimidad de castigar (Lawler y Bacharach, 1987). Esencialmente, el poder de una persona puede derivar de la autonomía de recusos procedente de la capacidad de intercambio que le brindan su posición y las alternativas ante las que se encuentra, o de las normas que regulan su papel en la estructura ${ }^{24}$. Así pues, el poder debería ser un candidato de excepción para examinar cómo interaccionan la cultura y la estructura.

En El príncipe, Maquiavelo sugiere que el poder es una capacidad desarrollada por un líder preparado para gobernar. Max Weber (1978: 926) define el poder como la capacidad de una persona o un grupo de llevar a cabo su voluntad a pesar de la resistencia que pueda encontrar por parte de otros actores. Estas dos aproximaciones sugieren que una persona poderosa concita al menos tres elementos: ocupar una posición estructural con una base de poder (como una posición de conexión y comunicación entre grupos independientes de una empresa), encontrarse en un entorno cultural basado en una cultura uniforme o en una cultura formada por grupos exclusivistas, y tener una personalidad que permite al líder sacar povecho de los recursos a su disposición. El poder es una capacidad que está determinada por la interpretación que los individuos

${ }^{24}$ Véase el trabajo de Coller (1997) para un análisis de las relaciones de intercambio en la empresa como fuente de poder. 
hacen de su situación y de su entorno. Tal interpretación se realiza a través de esquemas cognitivos ${ }^{25}$. La naturaleza construida, estereotipada, de esos esquemas pone de relieve el papel del factor cultural en la explicación del poder.

Por ejemplo, el poder de los Medici se configuró gracias a la confluencia de elementos estructurales y culturales (Padgett y Ansell, 1993). La combinación de una posición estructural de puente con una cultura que impedía a la burguesía mercantil alcanzar el estatus de la aristocracia permitió a los Medici aprovecharse de su posición supuestamente marginada para convertirse en una de las familias más poderosas de su tiempo. Visto así, el poder y su desarrollo son algo más que la mera suma del entorno cultural apropiado con la posición estructural oportuna. En definitiva, el poder es algo más que una coincidencia feliz de estos dos elementos. De aquí derivamos la proposición siguiente.

Proposición 2A. La capacidad de poder está ligada a posiciones con recursos (como el papel de puente y de autoridad), aunque su utilización estará modulada por las normas culturales sobre el uso del poder. El uso real del poder depende también de características personales, como la tolerancia al riesgo o a la agresión.

Más explícitamente, se puede suponer que la combinación de la posición estructural y la homogeneidad de normas culturales puede determinar qué posiciones de la estructura informal están mejor situadas para aprovechar sus recursos de poder. Las posiciones medias suelen estar bien situadas para aprovecharse de los conflictos normativos entre elites o para situarse en posiciones más centrales (Cook y Emerson, 1984). La heterogeneidad cultural sugiere la existencia de perspectivas múltiples, lo que genera más capacidad de estrategia y de negociación. Sin embargo, cuando la cultura de la organización es uniforme, homogénea, hay menos espacio para el disenso en los estratos más bajos de la organización ${ }^{26}$. En consecuencia, se puede plantear la proposición siguiente.

Proposition 2B. La naturaleza de la cultura determina si las posiciones mejor situadas para aprovechar sus recursos son las capas más altas o más bajas de la organización. La homogeneidad cultural debería reforzar el poder de las posiciones más altas, mientras que la heterogeneidad cultural refuerza el de las posiciones intermedias.

25 Sobre la noción de «esquema», su naturaleza cultural y su uso en las ciencias sociales, véanse los trabajos de DiMaggio (1997) y de Harris (1994). Véase el estudio de Guillén (1994) sobre la construcción de los esquemas cognitivos de las elites empresariales desde una perspectiva comparada.

${ }_{26}$ Ciertamente, esto lleva a plantear en primer lugar por qué la cultura es uniforme o heterogénea. 


\section{TURBULENCIA Y CAMBIO}

En esta sección se discuten las reacciones de la organización al cambio. Las culturas organizativas pueden ser más o menos resistentes a los cambios en la estructura y los objetivos de la organización. El cambio puede tener orígenes múltiples, como las fusiones con otras organizaciones, reestructuraciones, o los imprevistos en el entorno. Cualquier cambio en la organización amenaza con alterar la distribución de prestigio existente, lo que suele llevar a los miembros de la organización a resistirse a los cambios a pesar de que éstos se perciban como positivos para la organización en su conjunto ${ }^{27}$.

Incluso si la cultura de la organización es innovativa y efectiva, no se garantiza una adaptación al cambio con éxito. La información y los recursos son determinantes en los momentos de cambio y de crisis, y pueden ser movilizados fácil y rápidamente a través de las conexiones a lo largo de la estructura. Esto quiere decir que la capacidad de adaptación y de supervivencia de la organización depende en parte de aquellas personas cuyas decisiones están guiadas por puntos de vista apropiados y flexibles que se han construido a base de las informaciones y recursos provenientes de los distintos vínculos que mantienen con diferentes partes de la organización. De esta premisa se deriva la proposición siguiente.

Proposición 3A. La adaptación y la supervivencia de las organizaciones en condiciones de cambios turbulentos responden principalmente a la combinación de culturas innovadoras y externalizadoras. También dependen en gran medida de que existan vínculos organizativos que generen recursos y que hagan de puente entre puntos dispersos tanto dentro de la organización como entre organizaciones. Estos "puentes» permiten la transferencia tanto de información como de recursos. Por contra, la ausencia de estos elementos tiene sus consecuencias más negativas en momentos de cambio turbulento.

La legitimidad es la creencia generalizada de que una acción es válida y justificada: es aceptable por los demás, responde a sus expectativas, y se adecua a lo que es considerado normal y apropiado. La legitimidad de las acciones es en gran medida un fenómeno normativo que responde a una interpretación ex post facto que ningún actor suele controlar (Podolny, 1993). La cultura de la organización, a través de su manifestación en la identidad organizativa del actor, ofrece el tipo de legitimidad necesario para las acciones del individuo en su respuesta al cambio. Externamente, la legitimidad de las acciones y de las estructuras organizativas que se adecuan a las expectativas de otros actores proviene del entorno. Desde el punto de vista del análisis de redes, la legitimidad surge cuando los vínculos de un actor alcanzan a otros con identidades compa-

${ }^{27}$ Hay que tener presente que las jerarquías de prestigio surgen de la evaluación que las personas hacen teniendo en cuenta la posición relativa de cada uno y de los demás dentro de la organización (Podolny, 1993). 
tibles que interpretan el comportamiento y los símbolos del primero como apropiado. Pero del mismo modo en que el estatus puede prevenir la formación de vínculos (Podolny, 1993), la legitimidad (o, mejor dicho, su inexistencia) puede dificultar entrar en contacto con aquellos actores que pueden transferir legitimidad más fácilmente. La legitimidad, además de ser un tipo de evalución normativa, tiene también implicaciones estructurales. Internamente, la presencia o la falta de legitimidad delimitan las reacciones posibles de la organización al cambio.

Proposición 3B. Dado que la legitimidad emerge de interpretaciones de la acción y de la posible sanción que imponen otros, y dado que la legitimidad determina quiénes son los otros actores con los que uno/a se relaciona, la legitimidad o la falta de ella pueden limitar el repertorio de reacciones posibles a las situaciones de cambio. De esta manera puede resultar que las posibilidades de adaptación o de supervivencia al cambio se vean afectadas.

\section{LA DEFINICIÓN DE SIGNIFICADOS DENTRO DE LA ORGANIZACIÓN}

La definición de significados ha centrado la atención de las investigaciones sobre aspectos normativos de la realidad social. Los estudios culturales consideran que la interpretación individual de la sociedad (también llamada definición y atribución de significados) está guiada por el medio cultural en el que la gente se encuentra (Hirsch, 1986; Hirsch y Shanley, 1996). Los teóricos de la identidad han observado que la identidad contribuye a dotar de significado aquellos aspectos que se ven sujetos a cambios en el entorno (Gioia y Thomas, 1996). Hace ya tiempo que los científicos sociales vienen señalando el poder del lenguaje para controlar la definición de significados, o la construcción reflexiva e interpretativa compartida de acontecimientos una vez ya han ocurrido (Berger y Luckmann, 1986). Las retóricas alternativas, o los mitos en la organización, surgen como un intento de controlar la interpretación de la realidad (Olick y Levy, 1997) ${ }^{28}$. Esas retóricas suelen favorecer la adopción de la ideología de la organización o de la inevitabilidad del cambio, especialmente en esas personas que se benefician potencialmente de estos fenómenos. Al mismo tiempo, los mitos creados, las explicaciones dominantes, la definición de la realidad, suelen aportar un vocabulario que ayuda a explicar los acontecimientos traumáticos de forma agradable para aquellas personas afectadas negativamente por el cambio (Diamond, 1993).

${ }^{28}$ El concepto de mito aquí está despojado de su componente antropológico decimonónico y se aproxima a la definición sociológica moderna que entiende que un mito es una explicación de la realidad inmediata que expresa creencias colectivas de mayor o menor arraigo y que se manifiesta en diferentes formas de comunicación. 
Los análisis estructurales también han estudiado el proceso de definición de significados. Por ejemplo, Ibarra y Andrews (1993) consideran que las actitudes y opiniones favorables sobre la propia organización suelen estar influenciadas por la posición individual en la estructura informal. Distintos mecanismos relacionales operan en la formación de actitudes diferentes: la centralidad en la red de relaciones suele dar lugar a actitudes instrumentales, mientras que la proximidad genera actitudes afectivas.

Los mitos, las definiciones de la situación, la atribución de significados, no nacen de la nada. Por un lado, surgen en parte de las interacciones entre personas y luego se reifican e institucionalizan (Berger y Luckmann, 1986). Por otro lado, están también sujetas a la manipulación consciente de actores organizacionales clave (Bernstein, 1997). Los actores centrales sirven de filtro de información ya que tienen la capacidad de dar forma a la interpretación de los acontecimientos (la realidad). Tanto por su posición estructural como por su capacidad de crear interpretaciones (o alternativas a la interpretación), los actores centrales suelen compartir una definición de la realidad relativamente parecida en lo que respecta a la vida de la organización. Los mitos internalizados por actores centrales suelen ser principalmente los que reflejan los comportamientos organizacionales más legitimados y aceptados. La formación de ciertos grupos en la elite de la red (por ejemplo, cliques) puede quebrar la homogeneidad cultural que los actores centrales confieren a la organización, especialmente si esos grupos surgen como resultado de relaciones antagónicas (por ejemplo, los intentos de formación de «imperios locales» por parte de directivos).

Por contra, los actores marginales suelen estar expuestos a un nivel inferior de presiones para internalizar la definición de la realidad en la organización. Como estos actores ocupan posiciones que reciben pocos flujos de información, por definición, también tendrán menos presión para adoptar o creerse las definiciones de la realidad a las que están expuestos. Tienen mayor espacio para dar lugar a interpretaciones ajenas a los mitos centrales de la organización. Así pues, los actores marginales deberían desplegar un repertorio de mitos más amplio, y la internalización de esos mitos debería ser también más débil que la de los actores centrales.

Proposición 4. La adopción de retóricas interpretativas depende de la posición individual en la estructura social informal. Los actores centrales, como ocupantes de posiciones estructurales clave y como participantes activos en la creación de esas retóricas, deberían adoptar de forma más homogénea los mitos de la organización que legitiman su funcionamiento y sus objetivos. Una posición marginal en la red de relaciones debería reducir la probabilidad de incorporación de estos mitos. Se espera que los actores en dicha posición muestren una mayor variedad de interpretaciones sobre la realidad de la organización. Los actores marginales pueden incluso adoptar retóricas de oposición. Como su marginalidad reduce su solidaridad con otros miembros de la 
organización, los mitos de oposición constituirán unas bases débiles desde las que articular ningún tipo de acción.

\section{CONCLUSIÓN}

El argumento presentado se puede resumir en la siguiente proposición: la relación entre la estructura social y la cultura en una organización crea las condiciones en las que se desarrollan los comportamientos de los miembros. Este artículo ilustra el desarrollo de hipótesis empíricamente comprobables sobre el resultado de la interacción entre cultura organizativa y estructuras sociales en las organizaciones. Aunque nuestras proposiciones pueden generalizarse a espacios más amplios, nos limitamos al ámbito organizacional en parte porque los conceptos de estructura y cultura en este ámbito son más concretos, y en parte también por la gran cantidad de conocimiento acumulado sobre el comportamiento de las organizaciones y de sus miembros.

Si nuestros planteamientos son correctos, las investigaciones futuras no pueden obviar la relación entre estructura y cultura, sino que deben enfrentar esta cuestión teórica directamente ${ }^{29}$. Quizá una manera de aproximarse a esta cuestión es a través del renovado enfoque de la acción. Los individuos actúan $\mathrm{y}$, aunque esas acciones parezcan impredecibles en el plano individual, las ciencias sociales han podido explicarlas centrándose en los comportamientos agregados. Sin embargo, la relación entre los patrones sociales de comportamiento y su ocurrencia individual sigue siendo una cuestión abierta que ha derivado en dos problemas conocidos: la falacia ecológica y el error fundamental de atribución.

El enfoque estructural se basa siempre en las limitaciones que la posición de una persona en la estructura impone sobre sus opciones de acción. Del mismo modo, los argumentos culturales se centran en cómo los elementos culturales constriñen el comportamiento, llegando a generar en casos extremos una visión "hiper-socializada» del comportamiento humano (Wrong, 1961). Quizá la acción es el resultado de la combinación de exigencias fragmentadas y contradictorias de aspectos culturales y estructurales de la condición social de cada uno. Tal y como Simmel apuntó a principios del siglo Xx, la individualidad emerge de la necesidad de elegir, pero las opciones a elegir surgen de las condiciones culturales y estructurales en las que cada uno se encuentra.

29 Sobre las relaciones entre estructura, cultura y acción social, véase también el trabajo de Emirbayer y Goodwin (1994). 


\section{BIBLIOGRAFÍA}

AllCorn, S. (1995): «Understanding Organizational Culture as the Quality of Workplace Subjectivity", Human Relations, núm. 48, pp. 73-96.

ARONSON, Elliot (2000 [1972]): El animal social, Madrid: Alianza Editorial (8.a ed.).

Ashforth, B. E., y Mael, F. (1989): «Social Identity Theory and the Organization», Academy of Management Review, núm. 14, pp. 20-39.

Baron, J. N., y Pfeffer, J. (1994): "The Social Psychology of Organizations and Inequality», Social Psychology Quarterly, núm. 57, pp. 190-210.

Berger, Peter, y Luckmann, Thomas (1986): La construcción social de la realidad, Buenos Aires: Amorrortu.

Berkovwitz, S. D. (1982): An Introduction to Structural Analysis: The Network Approach to Social Research, Toronto: Buttesworth.

Berstein, M. (1997): "Celebration and Supression: The Strategic Uses of Identity by the Lesbian and Gay Movement», American Journal of Sociology, núm. 103, pp. 531-565.

Brass, D. J., y Burkhardt, M. E. (1992): «Centrality and Power in Organizations», en N. Nohira y R. Eccles (eds.), Networks and Organizations, Boston: Harvard University Press, pp. 191-215.

BURAWOY, Michael (1986): El consentimiento en la producción, Madrid: Ministerio de Trabajo y Seguridad Social.

Burke, P. J. (1997): "An Identity Model for Network Exchange», American Sociological Review, vol. 62, pp. 134-150.

Burrell, G., y Morgan, G. (1979): Sociological Paradigms and Organizational Analysis: Elements of the Sociology of Corporate Life, London: Heinemann.

BurT, R. S. (1980): «Models of Network Structure», Annual Review of Sociology, vol. 6, pp. 79141.

- (1992): Structural Holes: The Social Structure of Competition, Cambridge: Harvard University Press.

Cerulo, Karen A. (1997): «Identity Construction: New Issues, New Directions», Annual Review of Sociology, vol. 23, pp. 385-409.

Coller, Xavier (1996): "Managing Flexibility in the Food Industry. A Cross-National Comparative Case Study in European Multinational Companies», European Journal of Industrial Relations, vol. 2, núm. 2, julio, pp. 153-172.

- (1997): La empresa flexible. Un estudio sociológico del impacto de la flexibilidad en el proceso de trabajo, Madrid: CIS-Siglo XXI.

Coller, Xavier; Edwards, Tony, y ReEs, Chris (1999): «Difusión e isomorfismo en las organizaciones multinacionales», REIS, núm. 86, pp. 79-94.

COOK, Karen S., y EMERSON, Richard M. (1984): «Exchange Network and the Analysis of Complex Organizations, Research in the Sociology of Organizations, vol. 3, pp. 1-30.

Cook, Karen S., y Whitmeyer, J. M. (1992): «Two Approaches to Social Structure: Exchange Theory and Network Analysis», Annual Review of Sociology, vol. 18, pp. 109-127.

Deal, T. E., y Kennedy, A. A. (1982): Corporate Cultures, Reading (MA): Addison-Wesley.

Denison, D. R., y Mishra, A. K. (1995): «Towards a Theory of Organizational Culture and Effectiveness», Organization Science, vol. 6, pp. 204-223.

Diamond, M. A. (1991): «Dimensions of Organizational Culture and Beyond», Political Psychology, vol. 12, pp. 509-522.

- (1993): The Unconscious Life of Organizations: Interpreting Organizational Identity, Westport (CT): Quorum.

DiMaggio, Paul (1992): "Nadel's paradox revisited: Relational and Cultural Aspects of Organizational Structure», en N. Nohira y R. Eccles (eds.), Networks and Organizations, Boston: Harvard University Press, pp. 118-142.

- (1994): «Culture and Economy», en Neil SMelSER y R. Swedborg, The Handbook of Economic Sociology, Princeton: Princeton University Press.

- (1997): «Culture and Cognition», Annual Review of Sociology, vol. 23, pp. 263-287. 
Dutton, J. E.; Dukerich, J. M., y Harquail, C. V. (1994): «Organizational Images and Member Identification", Administrative Science Quarterly, vol. 39, pp. 239-263.

Edwards, Tony; Rees, Chris, y Coller, Xavier (1999): «Structure, Politics and the Diffusion of Employment Practices in Multinationals», European Journal of Industrial Relations, vol. 5, núm. 3, pp. 286-306.

Elsbach, K. D., y Kramer, R. M. (1996): «Members' Responses to Organizational Identity Threats: Encountering and Countering the Business Week Ratings", Administrative Science Quarterly, vol. 41, pp. 442-476.

Emirbayer, M., y Goodwin, J. (1994): «Network Analysis, Culture, and the Problem of Agency", American Journal of Sociology, vol. 99, pp. 1411-1454.

EVERED, Roger (1983): "The Language of Organizations: The Case of the Navy», en L. Pondy, P. Frost, G. Morgan y T. Dandridge (eds.), Organizational Symbolism, Greenwich: JAI.

GARMENDIA, José A. (1988): «La cultura de la empresa: una aproximación teórica y práctica», REIS, núm. 41, pp. 7-23.

- (1994): Tres culturas. Organización y recursos humanos, Madrid: ESIC.

Gartrell, C. D. (1987): «Network Approaches to Social Evaluation», Annual Review of Sociology, vol. 13, pp. 49-66.

GARVÍA, Roberto (1997): En el país de los ciegos: La ONCE desde una perspectiva sociológica, Barcelona: Hacer Editorial.

Giddens, Anthony (1984): The Constitution of Society. Outline of the Theory of Structuration, Berkeley: University of California Press.

GioiA, D. A., y Thomas, J. B. (1996): «Identity, Image, and Issue Interpretation: Sense Making During Strategic Change in Academia», Administrative Science Quarterly, vol. 41, pp. 370403.

Gouldner, Alvin W. (1954): Patterns of Industrial Bureaucracy, Nueva York: The Free Press.

Granovetter, Mark S. (1973): "The Strength of Weak Ties", American Journal of Sociology, vol. 78, pp. 1360-1380.

- (1978): "Threshold Models of Collective Behaviors», American Journal of Sociology, vol. 83, pp. $1420-1443$.

Gregory, K. L. (1983): "Native View Paradigms: Multiple Culture and Culture Conflicts in Organizations", Administrative Science Quarterly, vol. 28, pp. 359-376.

Guillén, M. (1994): Models of Management. Work, Authority, and Organization in Comparative Perspective, Chicago: Chicago University Press.

Harris, S. G. (1994): «Organizational Culture and Individual Sense-Making: A Schema-Based perspective», Organization Science, vol. 5, pp. 309-321.

Heckscher, C. C. (1995): White-Collar Blues: Management Loyalties in an Age of Corporate Restructuring, Nueva York: Basic Books.

Heimer, C. A. (1992): «Doing Your Work and Helping Your Friends: Universalistic Norms about Obligations to Particular Others in Networks», en N. Nohira y R. Eccles (eds.), Networks and Organizations, Boston: Harvard University Press, pp. 142-164.

Hirsch, P. M. (1986): «From Ambushes to Golden parachutes: Corporate Takeovers as an Instance of Cultural Framing and Institutional Integration», American Journal of Sociology, vol. 91, pp. 800-837.

Hirsch, P. M., y Shanley, M. (1996): "The Rhetoric of Boundaryless-Or, How the Newly Empowered Managerial Class Bought into Its Own Marginalization», en M. Arthur y D. Rousseau (eds.), The Boundaryless Career, Nueva York: Oxford University Press, cap. 13.

Hofstede, G. (1980): Culture's Consequences: International Differences in Work-Related Values, Beverly Hills: Sage.

IBARRA, Herminia (1992a): «Structural Aligments, Individual Strategies, and Managerial Action: Elements Toward a Network Theory of Getting Things Done», en N. Nohira y R. Eccles (eds.), Networks and Organizations, Boston: Harvard University Press, pp. 165-188.

- (1992b): «Homophily and Differential Returns: Sex Differences in Networks Structure and Access in an Advertising Firm", Administrative Science Quarterly, vol. 37, pp. 422-447. 
IbarRA, Herminia, y ANDrews, Steven B. (1993): «Power, Social Influence, and Sense Making: Effects of Network Centrality and Proximity on Employee Perceptions», Administrative Science Quarterly, vol. 38, pp. 277-303.

Johnson, C., y Ford, R. (1996): «Dependence Power, Legitimacy, and Tactical Choice», Social Psychology Quarterly, vol. 59, pp. 126-139.

Keesing, R. (1974): «Theories of Culture», Annual Review of Anthropology, vol. 3, pp. 73-97.

Kotter, J., y Heskett, J. (1992): Corporate Culture and Performance, Nueva York: Free Press.

KrackHardT, D. (1992): «The Strength of Strong Ties: The Importance of Philos in Organizations", en N. Nohira y R. Eccles (eds.), Networks and Organizations, Boston: Harvard University Press, pp. 216-239.

LaWler, E. J., y BaCharach, S. B. (1987): «Comparison of Dependence and Punitive Forms of Power", Social Forces, vol. 66, pp. 446-462.

LAWLer, E. J., y Yoon, J. (1996): «Commitment in Exchange Relations: Test of a Theory of Relational Cohesion", American Sociological Review, vol. 61, pp. 89-108.

LeVitT, B., y MArCH, James G. (1988): «Organizational Learning», Annual Review of Sociology, vol. 14, pp. 319-340.

Martin, J., y Siehl, C. (1983): «Organizational Culture and Counter-Culture: An Uneasy Symbiosis", Organizational Dynamics, vol. 12, pp. 52-64.

Mayhew, B. M. (1980): «Structuralism Versus Individualism: Part 1, Shadowboxing in the Dark», Social Forces, vol. 59, pp. 335-375.

Meyer, A. D. (1982): "Adapting to Environmental Jolts», Administrative Science Quarterly, vol. 27: 515-537.

Meyer, G. W. (1994): «Social Information Processing and Social Networks: A Test of Social Influence Mechanisms», Human Relations, vol. 47, pp. 1013-1048.

Miguel, Jesús M. de (1990): El mito de la sociedad organizada, Barcelona: Península.

- (2001): «De claustro a laboratorio: cambios en la Universidad como organización del conocimiento", en VV.AA., Estructura y cambio social. Libro homenaje a Salustiano del Campo, Madrid: CIS, pp. 1179-1207.

Nohira, N., y ECCles, R. (eds.) (1992): Networks and Organizations, Boston: Harvard University Press.

OlicK, J. K., y Levy, D. (1997): «Colective Memory and Cultural Constrain: Holocaust, Myth and Rationality in German Politics», American Sociological Review, vol. 62, pp. 921-936.

OrTIZ, Luis (2000): Convergencia o permanencia de los sistemas de relaciones laborales: reacción sindical a la introducción del trabajo en equipo en la industria del automóvil, Madrid: Centro de Estudios Avanzados en Ciencias Sociales.

Oтт, J. S. (1989): The Organizational Culture Perspective, Pacific Grove: Brooks/Cole.

Ouchi, W. G., y Wilkins, A. L. (1985): «Organizational Culture», Annual Review of Sociology, vol. 11, pp. 457-483.

Padgett, J. F., y Ansell, C. K. (1993): «Robust Action and the Rise of the Medici, 14001434", American Journal of Sociology, vol. 98, pp. 1259-1319.

PARSONS, Talcott (1959e [1945]): Essays in Sociological Theory. Revised Edition, Nueva York: The Free Press.

- (1966): El sistema social, Madrid: Alianza Universidad.

PÉREZ-DíAz, Víctor, y RodríGuez, Juan Carlos (2001): Educación superior y futuro en España, Madrid: Fundación Santillana.

Pfeffer, J. (1981): «Management as Symbolic Action: The Creation and Maintenance of Organizational paradigms", Research in Organizational Behavior, vol. 3, pp. 1-51.

Pfeffer, J., y SalanciK, G. (1978): The External Control of Organizations, Nueva York: Harper.

Podolny, J. M. (1993): «A Status-Based Model of Market Competition», American Journal of Sociology, vol. 98, pp. 829-872.

Podolny, J. M., y BARON, J. N. (1997): «Resources and Relationships: Social Networks and Mobility in the Workplace», American Sociological Review, vol. 62, pp. 673-693. 
Powell, Walter W., y Brantley, P. (1992): «Competitive Cooperation in Biotechnology: Learning through Networks?», en N. Nohira y R. Eccles (eds.), Networks and Organizations, Boston: Harvard University Press, pp. 366-394.

Powell, Walter W., y Smith-Doerr, L. (1994): «Networks and Economic Life», en Neil SmelSER y R. SWedborg, The Handbook of Economic Sociology, Princeton: Princeton University Press, pp. 368-402.

REQUena SANTOS, Félix (1989): «El concepto de red social», REIS, núm. 48, pp. 137-152.

- (1996): Redes sociales y cuestionarios, Madrid: CIS.

RodríGuez DíAZ, Josep A. (1993): «La sociología académica», REIS, núm. 64, pp. 175-200.

- (1995): Análisis estructural y de redes, Madrid: Centro de Investigaciones Sociológicas.

SCHEIn, E. H. (1985): Organizational Culture and Leadership, San Francisco: Jossey-Bass.

- (1990): «Organizational Culture», American Psychologist, vol. 45, pp. 109-119.

- (1996): "Culture: The Missing Concept in Organizational Studies», Administrative Science Quarterly, vol. 41, pp. 229-240.

Selznick, P. (1949): TVA and the Grass Roots: A Study in the Sociology of Organizations, Berkeley: University of California Press.

Siehl, C., y MARTin, J. (1990): «Measuring Organizational Culture: Mixing Qualitative and Quantitative Methods», en M. Jones, M. Moore y R. Snyder (eds.), Inside Organizations: Understanding the Human Dimensions, Newbury Park: Sage, pp. 79-103.

Simmel, Georg (1971): "Freedom and the Individual», en Georg Simmel, On Individuality and Social Forms, Chicago: University of Chicago Press, pp. 217-226.

Smelser, Neil, y Swedborg, R.: The Handbook of Economic Sociology, Princeton: Princeton University Press.

SmirCich, Lynda (1983): "Concepts of Culture and Organizational Analysis», Administrative Science Quarterly, vol. 28, pp. 339-358.

Suitor, J. J.; Wellman, B., y Morgan, D. L. (1997): «It's About Time: How, Why, and When Networks Change», Social Networks, vol. 19, pp. 1-7.

Tolbert, Pamela S., y Zucker, Lynne G. (1996): "Institutional Analyses of Organizations: Legitimated but Not Yet Institutionalized?», en Hardy, C.; Clegg, Stwart, y Nord, W. (eds.), Handbook of Organization Studies, Thousand Oaks: Sage, pp. 175-190.

Trice, H., y BeYER, J. (1984): «Studying Organizational Culture Through Rites and Ceremonies", Academy of Management Review, vol. 9, pp. 653-659.

Uheara, E. (1990): «Dual Exchange Theory, Social Networks, and Informal Social Support», American Journal of Sociology, vol. 96, pp. 521-557.

VAn MaAnen, J., y Barley, S. R. (1984): "Occupational Communities: Culture and Control in Organizations", Research in Organizational Behavior, vol. 6, pp. 287-365.

Wasserman, S., y Faust, K. (1994): Social Network Analysis: Methods and Applications, Nueva York: Cambridge University Press.

Weber, Max (1978): Economy and Society. An Outline of interpretive sociology, Berkeley: University of California Press.

Welbourne, T. M., y Andrews, A. O. (1996): «Predicting the Performance of Initial Public Offerings: Should Human Resource Management Be in the Equation?», Academy of Management Journal, vol. 39, pp. 891-919.

White, H. C. (1992): Identity and Control: A Structural Theory of Social Action, Princeton: Princeton University Press.

Wilkins, A. L., y OuCHI, W. G. (1983): «Efficient Cultures: Exploring the Relationship between Culture and Organizational Performance», Administrative Science Quarterly, vol. 28, pp. 468-481.

Wrong, D. H. (1961): «The Oversocialized Conception of Man», American Sociological Review, vol. 26, pp. 184-193. 


\begin{abstract}
The interaction between culture and the informal structure of organization has diverse effects on the daily life of institutions. Some of them are the development of a certain organizational identity and the appearance of relationships of informal power. This paper puts forward some lines of research into these two aspects, taking advantage of some of the contributions of the analysis of networks. The authors venture to suggest some work hypotheses that can help to give direction to empirical research in the future.
\end{abstract}

